\title{
Análise das Variáveis Intervenientes na Tomada de Decisões Éticas dos Profissionais e Estudantes de Ciências Contábeis Brasileiros
}

\section{Analysis of the Variables Involved in the Ethical Decision Making of Professionals and Students of Brazilian Accounting Sciences}

\author{
Cristina Laidmera ; Alexandre André Fei1*b

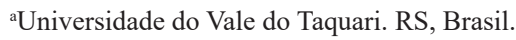 \\ bUniversidade do Vale do Taquari, Programa de Pós-Graduação Stricto Sensu em Sistemas Ambientais Sustentáveis, RS, Brasil. \\ *E-mail: alexandre.feil1@gmail.com
}

\begin{abstract}
Resumo
O objetivo geral é analisar os fatores que influenciam essa tomada de decisão, com base nos estudos conduzidos no Brasil. O presente estudo se utiliza da abordagem mista ou qualiquantitativa e os procedimentos técnicos adotados o classificam como revisão sistemática com metanálise. A revisão sistemática identificou 28 estudos conduzidos no Brasil sobre o tema, abrangendo 7.580 respondentes de 22 Estados das cinco regiões geográficas do país, no período compreendido entre os anos de 2006 e 2017. Os resultados indicam predomínio de estudos abordando as variáveis individuais, com destaque para o gênero, e entre as variáveis situacionais se destaca, por sua frequência, o Código de Ética Profissional do Contador - CEPC. Quanto à influência das variáveis, na decisão ética, no conjunto dos estudos analisados, as variáveis individuais obtiveram resultados mistos, à exceção da educação ética, a qual apresentou consenso sobre sua influência positiva. A metanálise indicou relação estatisticamente significativa da variável situacional CEPC com a tomada de decisão ética. Destacam-se também os componentes da variável intensidade moral da questão ética como campo em expansão e com resultados consistentes.
\end{abstract}

Palavras-chave: Ética Profissional. Variáveis Intervenientes. Metanálise.

\begin{abstract}
The general objective is to analyze the factors that influence this decision-making, based on the studies conducted in Brazil. The present study uses the mixed or qualitative and quantitative approach and the adopted technical procedures classify it as a systematic review with meta-analysis. The systematic review identified 28 studies conducted in Brazil on the subject, covering 7,580 respondents from 22 states in the five geographic regions of the country, in the period between 2006 and 2017. The results indicate a predominance of studies addressing the individual variables, for the gender, and among the situational variables, it is stood ut by its frequency the Professional Code of Ethics of the Accountant - CEPC. Regarding the variables influence in the ethical decision, in the set of studies analyzed, the individual variables obtained mixed results, except for ethical education, which presented a consensus about its positive influence. The meta-analysis indicated a statistically significant relationship of the situational variable CEPC with the ethical decision-making. The components of the moral intensity variable of the ethical question as a field in expansion and with consistent results are also noteworthy.
\end{abstract}

Keywords: Professional Ethics. Intermediate Variables. Meta-Analysis.

\section{Introdução}

Os escândalos e fraudes em corporações internacionais como a Enron (BBCBrasil, 2002), Worldcom (HORTA, 2002) e Xerox (BBCBrasil, 2003) têm sido retratados nos noticiários nacionais e internacionais. O envolvimento de profissionais da contabilidade nessas notícias tem gerado o descrédito da população relativamente à profissão contábil (ANZEH; ABED, 2015; MORAES; SILVA; CARVALHO, 2010) e ampliado o interesse sobre o papel destes profissionais nesses acontecimentos (LUPION, 2016).

A contabilidade objetiva fornecer informações úteis a diversos stakeholders para capacitá-los para tomada de decisões (MORAES; SILVA; CARVALHO, 2010). Esses autores ainda salientam que as informações contábeis geram consequências diretas na atividade econômica e, por essa razão, é necessário que os profissionais contábeis tenham consciência de suas responsabilidades.

Os comportamentos antiéticos de profissionais contábeis, além de causarem prejuízos ao sistema econômico (ANZEH; ABED, 2015), também promovem dano à imagem da categoria, visto que a conduta ética é essencial à preservação de uma profissão (MORAES; SILVA; CARVALHO, 2010). Sá (2012) enfatizando que, assim como a atitude correta dos profissionais enobrece uma profissão, o descumprimento dos princípios éticos resulta na sua desmoralização.

O estudo da ética profissional se divide em: a) ética normativa: apoia-se na abordagem filosófica e teológica da moral e fornece orientações sobre como os indivíduos deveriam agir; e b) ética descritiva ou empírica: busca explicar e prever o efetivo comportamento dos sujeitos diante de dilemas éticos (O’FALLON; BUTTERFIELD, 2005).

Os estudos realizados com o objetivo de estabelecer os fatores, que têm influência na tomada de decisões éticas dos profissionais, têm apresentado resultados distintos. O desenvolvimento cognitivo moral, apresentado pela teoria de Kohlberg (1969), é considerado como um dos fatores que tem 
influência sobre a tomada de decisões éticas (ALVES, 2005; FERRELL; FRAEDRICH; FERRELL, 2001; TREVINO, 1986). Connelly, Helton-Fauth e Mumford (2004) afirmam que as emoções positivas ou negativas, bem como sua intensidade, exercem influência sobre as escolhas éticas. Curtis (2006) aponta que o humor interfere na decisão dos profissionais de denunciar atitudes antiéticas. Cullinan et al. (2006) afirmam que indivíduos com mais alto grau de comprometimento, em sua atividade profissional, têm menor probabilidade de agir de forma antiética, independente se suas atitudes tragam benefício ou prejuízo à empresa.

Nesse contexto, este estudo objetiva analisar os fatores que influenciam a tomada de decisões éticas dos profissionais e estudantes brasileiros de contabilidade. A justificativa se centra na crescente preocupação, tanto para os profissionais quanto para os educadores, em desenvolver o raciocínio e o comportamento ético dos profissionais da contabilidade (SORENSEN; MILLER; CABE, 2015). O aumento da atenção acadêmica sobre o processo da tomada de decisões éticas, tanto pelos estudantes quanto pelos profissionais, é necessário para reverter o avanço dos casos de comportamento antiético (MORAES; SILVA; CARVALHO, 2010).

\section{Material e Métodos}

\subsection{Classificação da pesquisa}

A pesquisa se classifica como qualiquantitativa, pois a qualitativa explora os fenômenos em profundidade e seus benefícios incluem a extensão, a riqueza interpretativa e a contextualização dos fenômenos (SAMPIEIRI; COLLADO; LUCIO, 2013). Esses autores (p. 26) descrevem que a quantitativa: “[...] utiliza a coleta de dados para testar hipóteses, baseando-se na medição numérica e na análise estatística para estabelecer padrões e comprovar teorias". Nesse sentido, este estudo abordou uma revisão extensiva de um conjunto de estudos, a contextualização e a organização de seus achados e a apresentação de suas conclusões e características relevantes; além disso, foram aplicadas técnicas estatísticas para a análise conjunta dos resultados dos estudos por meio de uma metanálise.

Os procedimentos técnicos se centram na revisão sistemática e na metanálise. A revisão sistemática “[...] é uma forma de pesquisa que utiliza como fonte de dados a literatura sobre determinado tema" para avaliar sua consistência e a generalização (SAMPAIO; MANCINI, 2007, p. 84). Esses autores ainda enfatizam que algumas revisões sistemáticas podem incluir a síntese estatística dos resultados dos estudos por meio da metanálise, o que aumenta a amostra total e a precisão da estimativa. A metanálise é “[...] um tipo de análise de dados em que os resultados de vários estudos que abordam a mesma questão de pesquisa são combinados, gerando assim, estimativas que resumem o todo, chamadas de estimativas metanalíticas" (RODRIGUES, 2010, p.9).

\subsection{Unidade de análise e coleta dos dados}

A unidade de análise do presente estudo consiste nos resultados de pesquisas do tipo survey conduzidas no Brasil, com estudantes e profissionais contábeis, sobre o tema da ética profissional. A escolha da unidade de análise se justifica pelo crescente interesse sobre o aspecto da ética dos profissionais de contabilidade. Os critérios estabelecidos para inclusão na unidade de análise foram: pesquisas realizadas no Brasil, tendo como amostra estudantes e profissionais de contabilidade e que pesquise as variáveis intervenientes na tomada de decisão ética desses indivíduos no âmbito profissional. A coleta das pesquisas ocorreu em duas etapas; na primeira se utilizou a base de dados de periódicos e na segunda o processo snowball.

As bases de dados de periódicos utilizadas compreendem o Google Scholar, SciELO, Portal de Periódicos Capes e Spell, em função de serem as principais fontes de pesquisas científicas, em frequência de uso e número de estudos indexados. No Google Scholar foram inseridos na caixa de pesquisa os termos e expressões: ética profissional, decisão ética, contabilidade, questionário, survey e teste. A pesquisa retornou com cerca de 1900 resultados, entre os quais, após a leitura dos títulos e resumos, foram selecionados 23 estudos que atendiam aos critérios estabelecidos.

$\mathrm{Na}$ base de dados do Scielo se utilizaram as expressões: ética profissional, decisão ética e contabilidade e retornou com nove resultados, os quais foram lidos e enquadrados na unidade de análise. No Portal de Periódicos Capes foi inserida a expressão: ética profissional e contabilidade. Esta busca retornou 20 resultados, dos quais, excluindo-se um que já havia sido selecionado a partir do Scholar e os que não atendiam aos critérios de inclusão, não se agregaram novos resultados à unidade de análise.

No repositório Spel, foi inserida a expressão "ética profissional" e retornaram 50 resultados, deste total, quatro já integravam a unidade de análise e os demais não se enquadravam nos critérios de inclusão. Após a busca nos repositórios, foi realizada a pesquisa nas referências dos estudos selecionados, denominada de snowball, a fim de localizar outras pesquisas, que atendessem aos critérios de inclusão e que não tivessem sido listadas nas buscas supracitadas. Entretanto, esse procedimento não agregou outros estudos à unidade de análise.

A coleta ocorreu entre fevereiro e março de 2018 e obteve como resultado estudos concentrados em um intervalo temporal de 11 anos, entre 2006 e 2017. O gráfico da Figura 1 explicita o detalhamento dos estudos quanto ao número de respondentes por estudo. 
Figura 1 - Unidade de análise

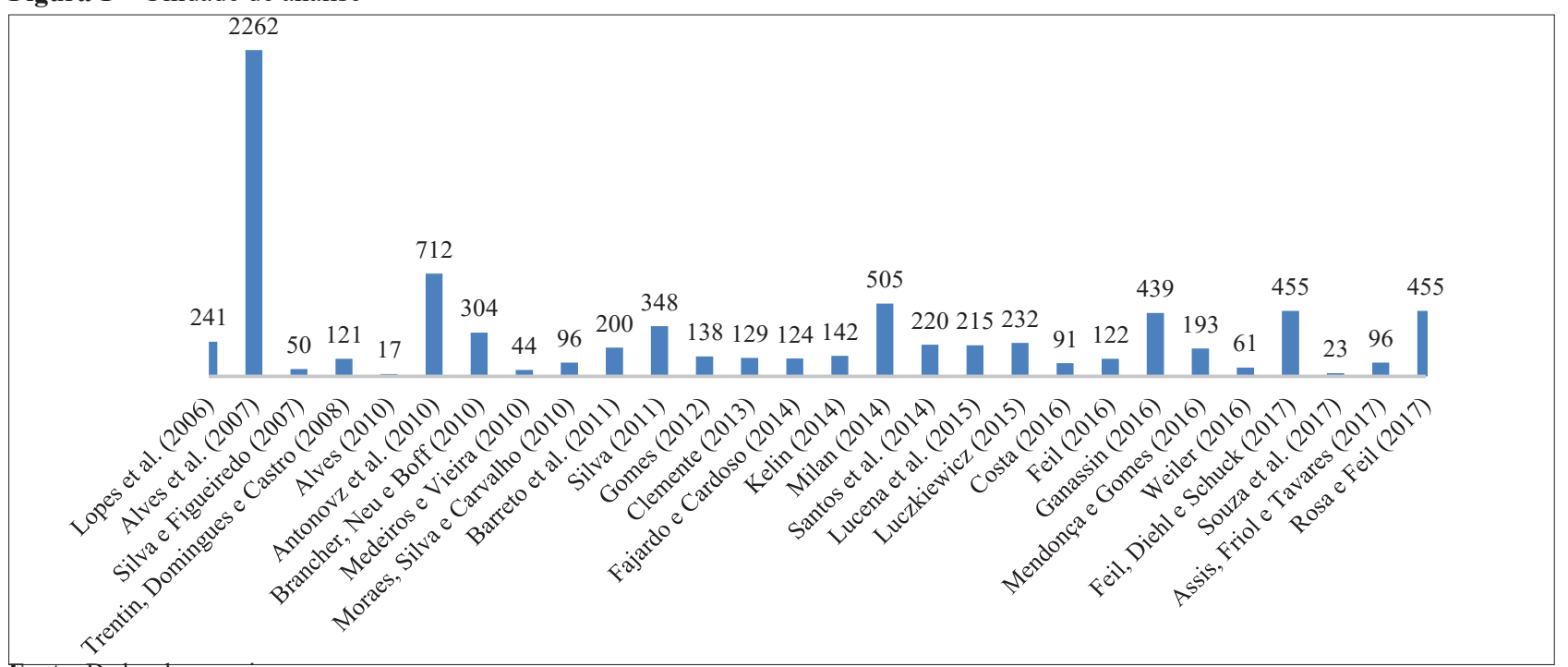

Fonte: Dados da pesquisa.

Essas informações demonstram a abrangência geográfica das amostras das pesquisas coletadas e informam que 22 Estados brasileiros estão representados neste estudo. Nesse sentido, a unidade de análise deste estudo totaliza 7.580 respondentes, entre profissionais contábeis e estudantes de Ciências Contábeis das cinco regiões geográficas do Brasil.

\subsection{Análise dos dados}

Após a localização dos estudos, que compõem a unidade de análise, os mesmos foram registrados em planilhas eletrônicas para organização preliminar das informações. Assim, em cada linha da planilha foi alocado um dos estudos, sobre os quais foram inseridas nas colunas as informações: a) autor; b) ano; c) procedimento de coleta de dados; d) tipo de publicação; e) periódico/evento/instituição; f) número de respondentes; g) local de coleta; h) número de profissionais; i) número de estudantes; j) variáveis analisadas; e k) achados.

A partir da organização preliminar dos dados, foram elaboradas novas planilhas, de modo a agrupar e detalhar aspectos particulares de cada pesquisa e evidenciar informações a partir do conjunto das mesmas. Tais informações foram analisadas por meio de revisão sistemática. Os estudos que apresentaram dados de análise estatística foram, posteriormente, objeto de metanálise por meio do teste nãoparamétrico Qui-Quadrado. O teste Qui-Quadrado é utilizado para comprovar se existem diferenças significativas entre duas distribuições (BISQUERRA; SARRIERA; MARTÍNEZ, 2004) e não se baseia em dados contínuos normalmente distribuídos (FIELD, 2009).

\section{Resultados e Discussão}

\subsection{Revisão sistemática}

A revisão sistemática identificou 28 estudos conduzidos no Brasil sobre o tema, abrangendo 7.580 respondentes de 22 Estados das cinco regiões geográficas do país, ao longo do período entre os anos de 2006 e 2017. Tal abrangência demonstra que o interesse pelo tema da tomada de decisão ética dos profissionais e estudantes de Ciências Contábeis é crescente entre pesquisadores e instituições de todo o país. A elevada titulação acadêmica da maioria dos autores e a publicação dos estudos em periódicos dos estratos superiores da classificação Qualis, além de evidenciar o interesse pelo tema, aumentam a credibilidade relativa à qualidade científica dos estudos.

Quanto à frequência de abordagem das variáveis, este estudo verificou que as pesquisas nacionais se dedicam, predominantemente, às variáveis individuais, em comparação às variáveis situacionais e às relativas à questão ética. Isto pode se dever, entre outras razões, ao fato de que estas são mais diretamente verificáveis e mensuráveis em um survey do que as que envolvem alguns dos fatores situacionais ou as ligadas à questão ética.

As variáveis individuais investigadas, com maior frequência, nos estudos em análise foram o gênero $(53,6 \%)$, a idade (46,4\%), a maturidade acadêmica $(39,3 \%)$, a experiência profissional $(32,1 \%)$ e a religiosidade $(32,1 \%)$. Estudos correlatos também apresentam o gênero como a variável mais frequente (LOE et al., 2000; O'FALLON; BUTTERFIELD, 2005).

A revisão sistemática revelou que há predomínio de artigos publicados em periódicos (14), seguido pelas pesquisas em nível de graduação (5), dissertações de mestrado (5) e publicados em eventos (4). A distribuição dos estudos entre diversos tipos de publicações demonstra que o tema da tomada de decisão ética profissional é de amplo interesse para os profissionais contábeis e, também, para a reflexão sobre as práticas educacionais direcionadas à ética profissional.

A região geográfica dos periódicos que publicaram os artigos se centra na região Sudeste do país (64,3\%), na região Nordeste $(14,3 \%)$ e as regiões Sul, Centro-oeste e Norte contam cada uma com 7,1\%. (Quadro 1). 
Quadro 1 - Artigos, periódicos e dados de publicação

\begin{tabular}{|c|c|c|c|c|c|c|c|}
\hline$\stackrel{0}{\stackrel{0}{e}} \stackrel{0}{Z}$ & ف & $\stackrel{\frac{n}{\tilde{E}}}{\tilde{\sigma}}$ & 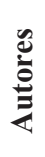 & : & $\underset{j}{\stackrel{0}{e}}$ & 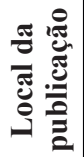 & 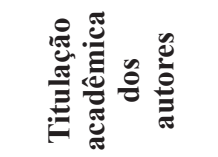 \\
\hline Lopes et al. (2006) & $\begin{array}{l}\text { Ensaio: Avaliação e políticas públicas em } \\
\text { educação }\end{array}$ & B1 & 4 & 6 & $\begin{array}{c}\text { Fundação } \\
\text { CESGRANRIO }\end{array}$ & RJ & $\begin{array}{c}1 \text { Pós-Doutor, } 3 \\
\text { Doutores }\end{array}$ \\
\hline Alves et al. (2007) & Revista de Contabilidade e Finanças - USP & B4 & 4 & 38 & Pública & SP & 4 Doutores \\
\hline $\begin{array}{c}\text { Silva e Figueiredo } \\
(2007)\end{array}$ & Revista Brasileira de Contabilidade & $\mathrm{C}$ & 2 & 5 & Outro - CFC & $\mathrm{DF}$ & $\begin{array}{l}1 \text { Mestre, } 1 \\
\text { Graduanda }\end{array}$ \\
\hline $\begin{array}{l}\text { Antonovz et al. } \\
(2010)\end{array}$ & $\begin{array}{c}\text { Revista de Contabilidade e Organizações } \\
\text { - USP }\end{array}$ & A2 & 4 & 8 & Pública & SP & $\begin{array}{l}3 \text { Doutoras, } 1 \\
\text { Mestre }\end{array}$ \\
\hline $\begin{array}{l}\text { Brancher, Neu e } \\
\text { Boff }(2010)\end{array}$ & Unoesc \& Ciência - ACSA & B4 & 3 & 5 & Comunitária & $\mathrm{SC}$ & $\begin{array}{l}1 \text { Mestre, } 2 \\
\text { Graduandas }\end{array}$ \\
\hline $\begin{array}{c}\text { Medeiros e Vieira } \\
(2010)\end{array}$ & Revista Interface - UFRN & B3 & 2 & - & Pública & $\mathrm{RN}$ & $\begin{array}{l}1 \text { Mestre, } 1 \\
\text { Doutora }\end{array}$ \\
\hline $\begin{array}{l}\text { Moraes, Silva e } \\
\text { Carvalho (2010) }\end{array}$ & Pensar Contábil & B2 & 3 & 9 & Outro - CRC/RJ & RJ & $\begin{array}{l}1 \text { Doutor, } 2 \\
\text { Mestres }\end{array}$ \\
\hline $\begin{array}{l}\text { Barreto et al. } \\
\qquad(2011)\end{array}$ & ReCont: Registro Contábil - UFAL & B5 & 4 & 3 & Pública & $\mathrm{AL}$ & $\begin{array}{c}2 \text { Pós-Doutores, } \\
1 \text { Doutor, } 1 \\
\text { Mestre }\end{array}$ \\
\hline $\begin{array}{c}\text { Fajardo e Cardoso } \\
(2014) \\
\end{array}$ & Brazilian Business Review - FUCAPE & A2 & 2 & - & Privada & ES & 2 Doutores \\
\hline $\begin{array}{l}\text { Lucena et al. } \\
\quad(2015)\end{array}$ & $\begin{array}{c}\text { Revista de Contabilidade e Organizações } \\
\text { - USP }\end{array}$ & A2 & 4 & 1 & Pública & SP & 4 Doutores \\
\hline $\begin{array}{c}\text { Mendonça e } \\
\text { Gomes (2016) }\end{array}$ & $\begin{array}{l}\text { Revista de Auditoria, Governança e } \\
\text { Contabilidade }\end{array}$ & B4 & 2 & - & Privada & MG & $\begin{array}{l}1 \text { Mestre, } 1 \\
\text { Graduando }\end{array}$ \\
\hline $\begin{array}{c}\text { Feil, Diehl e } \\
\text { Schuck (2017) } \\
\end{array}$ & Cad. EBAPE.BR - FGV & A2 & 3 & - & Privada & RJ & $\begin{array}{c}2 \text { Doutores, } 1 \\
\text { Mestre }\end{array}$ \\
\hline Rosa e Feil (2017) & $\begin{array}{l}\text { Revista Científica On-Line - Tecnologia - } \\
\text { Gestão - Humanismo }\end{array}$ & B4 & 2 & - & Pública & SP & $\begin{array}{l}1 \text { Doutor, } 1 \\
\text { Graduanda }\end{array}$ \\
\hline Souza et al. (2017) & $\begin{array}{l}\text { Revista de Administração e Contabilidade } \\
\text { da Faculdade Estácio do Pará - Belém }\end{array}$ & B4 & 5 & - & Privada & PA & $\begin{array}{l}1 \text { Doutor, } 4 \\
\text { Graduandas }\end{array}$ \\
\hline
\end{tabular}

Fonte: Dados da pesauisa.

O ano de 2010 conta com o maior número de publicações (28,6\% dos artigos), seguido por 2017 (21,4\% dos artigos). Destaca-se que $28,6 \%$ dos artigos foram publicados em periódicos com classificação Qualis A2 da área Administração pública e de empresas, Ciências Contábeis e Turismo; 7,1\% em periódicos com classificação $\mathrm{B} 1$ e $7,1 \%$ em periódicos classificados no nível B2. Nesse sentido, 42,8\% dos artigos foram publicados em periódicos avaliados nos estratos superiores da classificação Qualis, mantida e atualizada anualmente pela Coordenação de Aperfeiçoamento de Pessoal de Nível Superior (CAPES). Outros 57,1\% dos artigos componentes desta unidade de análise foram publicados em periódicos de classificação Qualis B3 e inferiores.

Os artigos possuem, em média, 3,14 autores; acerca da titulação acadêmica dos autores, a maioria dos autores é composta de Doutores (52,3\%), 20,4\% são Mestres, 20,4\% são graduandos e 6,8\% de Pós-Doutores. Essa distribuição indica que profissionais de elevada titulação acadêmica têm se dedicado ao tema, o que é positivo, pois sugere a produção de pesquisas de alto rigor e nível científico.

As cinco dissertações de mestrado sobre o tema também provêm de três regiões geográficas distintas e de IES públicas e privadas. As apresentações desses estudos se distribuíram entre 2011 e 2016 (Quadro 2).

Quadro 2 - Trabalhos de Conclusão de Curso (TCC) e dissertações

\begin{tabular}{|c|c|c|c|c|}
\hline Autor & Tipo & Instituição & $\begin{array}{c}\text { Natureza } \\
\text { administrativa }\end{array}$ & Região \\
\hline Alves (2010) & TCC & Universidade Federal da Grande Dourados & Pública, Federal & Centro-Oeste \\
\hline Gomes (2012) & TCC & Universidade Estadual da Paraíba & Pública, Estadual & Nordeste \\
\hline Kelin (2014) & TCC & Universidade de Caxias do Sul & Privada, Comunitária & Sul \\
\hline Costa (2016) & TCC & Universidade Tecnológica Federal do Paraná & Pública, Federal & Sul \\
\hline Weiler (2016) & TCC & Universidade do Vale do Taquari & Privada & Sul \\
\hline Silva (2011) & Dissertação & Universidade Presbiteriana Mackenzie & Privada, Confessional & Sudeste \\
\hline Clemente (2013) & Dissertação & Universidade Presbiteriana Mackenzie & Privada, Confessional & Sudeste \\
\hline Milan (2014) & Dissertação & Fundação Escola de Comércio Álvares Penteado & Privada & Sudeste \\
\hline Luczkiewicz (2015) & Dissertação & Fundação Escola de Comércio Álvares Penteado & Privada & Sudeste \\
\hline Ganassin (2016) & Dissertação & $\begin{array}{c}\text { Universidade de Brasília, Universidade Federal da } \\
\text { Paraíba e UFRN }\end{array}$ & Públicas, Federais & $\begin{array}{c}\text { Centro-Oeste e } \\
\text { Nordeste }\end{array}$ \\
\hline
\end{tabular}

Fonte: Dados da pesquisa. 
A concentração de estudos de 2010 a 2016 denota uma preocupação crescente com as questões éticas no âmbito da profissão contábil, a qual pode ter sido desencadeada pela crescente atenção dada pela mídia e pela comunidade acadêmica a fraudes em corporações (COSTA, 2011; ELY, 2010; FILGUEIRAS, 2015; RAMACCIOTTI, 2007). O Quadro 3 apresenta as literaturas publicadas em eventos.

Quadro 3 - Pesquisas apresentadas em eventos

\begin{tabular}{|c|c|}
\hline Autor & Evento \\
\hline $\begin{array}{c}\text { Trentin, Domingues } \\
\text { e Castro (2008) }\end{array}$ & $\begin{array}{c}\text { XV Congresso Brasileiro de Custos - } \\
\text { Curitiba/PR }\end{array}$ \\
\hline Santos et al. (2014) & $\begin{array}{c}\text { XVII SemeAd - Seminários em } \\
\text { Administração - São Paulo/SP }\end{array}$ \\
\hline Feil (2016) & $\begin{array}{c}20^{\circ} \text { Congresso Brasileiro de } \\
\text { Contabilidade - Fortaleza/CE }\end{array}$ \\
\hline Assis, Friol e & $13^{\circ}$ Encontro Nordestino de \\
Tavares (2017) & Contabilidade - Natal/RN \\
\hline
\end{tabular}

Fonte: Dados da pesquisa.
As publicações em eventos relacionadas à tomada de decisão ética dos profissionais contábeis se apresentam em menor número. Em suma, pode-se notar que os estudos sobre as variáveis intervenientes na decisão ética de estudantes e de profissionais contábeis vêm sendo conduzidos e publicados em todas as regiões do país, por instituições de diversas naturezas administrativas. Também se evidencia uma tendência de crescimento do número de pesquisas sobre o tema ao longo dos anos, o que indica que o interesse pelo mesmo ainda está em evolução no Brasil, a exemplo de outros países (KISHGEPHART; HARRISON; TREVINO, 2010, LOE et al., 20005, O'FALLON; BUTTERFIELD, 2005).

Além dessas informações da literatura sobre a unidade de análise, foi elaborado um quadro de resultados dos estudos em relação aos fatores intervenientes, que influem na tomada de decisão ética dos profissionais e estudantes contábeis (Quadro 4).

Quadro 4 - Estudos com análise das variáveis intervenientes na tomada de decisão ética

\begin{tabular}{|c|c|c|c|c|c|c|c|c|c|c|c|c|c|c|}
\hline & \multicolumn{2}{|c|}{ Amostra } & \multirow[b]{2}{*}{ 岇 } & \multirow[b]{2}{*}{ 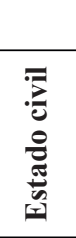 } & \multicolumn{8}{|c|}{ Variáveis individuais } & \multirow{2}{*}{\begin{tabular}{|c|} 
CEPC \\
\\
U⿱宀匕 \\
\end{tabular}} & \multirow{2}{*}{ 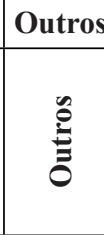 } \\
\hline Autor e Ano & 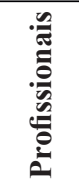 & 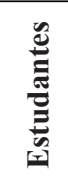 & & & 递 & 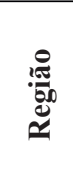 & 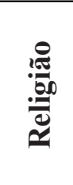 & 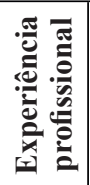 & 冚 & 冚 & 芯 & $\stackrel{\mathscr{E}}{E}$ & & \\
\hline Assis, Friol e Tavares (2017) & 96 & & $\mathrm{BR}$ & & $\mathrm{Sim}$ & Não & & & & & & & & \\
\hline Luczkiewicz (2015) & & 232 & 8 & & Não & Sim & Não & Não & Sim & Não & & Não ${ }^{1}$ & & \\
\hline Lopes et al. (2006) & & 241 & - & & $\mathrm{Sim}$ & & & & Sim & & & & & $\mathrm{Sim}^{9}$ \\
\hline $\begin{array}{l}\text { Moraes, Silva e Carvalho } \\
(2010)\end{array}$ & & 96 & RJ & & Sim & & & Sim & & Sim & & & & \\
\hline Silva (2011) & & 348 & SP & & $\mathrm{Sim}$ & & & Sim & & $\mathrm{Sim}$ & Sim & & & \\
\hline Clemente (2013) & 129 & & - & & Não & & Não & & & Não & & $\mathrm{Não}^{2}$ & & $\mathrm{Não}^{10}$ \\
\hline Fajardo e Cardoso (2014) & 124 & & - & Não & Não & & & & & Não & & $\mathrm{Não}^{3}$ & & $\operatorname{Sim}^{11}$ \\
\hline Milan (2014) & & 505 & SP & & Não & & Não & Sim & Sim & Não & & $\mathrm{Sim}^{4}$ & & \\
\hline Lucena et al. (2015) & 215 & & - & & Não & & & & & Não & & $\operatorname{Sim}^{5}$ & & \\
\hline Costa (2016) & & 91 & PR & & Não & & & Não & Não & Não & & & & \\
\hline Feil (2016) & 122 & & $\mathrm{RS}$ & & Não & & Sim & Não & Não & Não & & & Sim & \\
\hline Ganassin (2016) & & 439 & DF & & Não & & Não & Não & & $\mathrm{Sim}$ & & & Não & \\
\hline Mendonça e Gomes (2016) & & 193 & $\mathrm{MG}$ & Não & $\mathrm{Sim}$ & & $\mathrm{Sim}$ & & Sim & Não & & Não ${ }^{6}$ & & \\
\hline Feil, Diehl e Schuck (2017) & & 455 & $\mathrm{RS}$ & & $\mathrm{Sim}$ & & $\mathrm{Sim}$ & Sim & Sim & $\mathrm{Sim}$ & Sim & $\operatorname{Sim}^{7}$ & & \\
\hline Weiler (2016) & 61 & & $\mathrm{RS}$ & & & & $\mathrm{Sim}$ & & & $\mathrm{Sim}$ & & & & \\
\hline Gomes (2012) & 138 & & - & & & & & Sim & Sim & & & & $\mathrm{Sim}$ & \\
\hline Barreto et al. (2011) & 200 & & $\mathrm{PE}$ & & & & Não & & & & & & & \\
\hline $\begin{array}{l}\text { Trentin, Domingues e Castro } \\
(2008)\end{array}$ & & 121 & $\mathrm{SC}$ & & & & & & & & Sim & & & \\
\hline Brancher, Neu e Boff (2010) & & 304 & $\mathrm{SC}$ & & & & & & $\mathrm{Sim}$ & & Sim & & & \\
\hline Rosa e Feil (2017) & 455 & & $\mathrm{RS}$ & & & & & & & & Sim & & Sim & \\
\hline Alves et al. (2007) & 2262 & & $\mathrm{BR}$ & & & & & & & & & & $\mathrm{Sim}$ & \\
\hline Alves (2010) & 17 & & MS & & & & & & & & & & Sim & \\
\hline Kelin (2014) & & 142 & $\mathrm{RS}$ & & & & & & & & & & Sim & \\
\hline Souza et al. (2017) & 23 & & PA & & & & & & & & & & Sim & \\
\hline Silva e Figueiredo (2007) & & 50 & $\mathrm{CE}$ & & & & & & Sim & & & & & \\
\hline Medeiros e Vieira (2010) & & 44 & $\mathrm{RN}$ & & & & & & Não & & & & & \\
\hline Santos et al. (2014) & 220 & & $\mathrm{MG}$ & & & & & & & & & & & $\operatorname{Sim}^{12}$ \\
\hline Antonovz et al.(2010) & 234 & 478 & PR & & Sim & & & & & & & & & \\
\hline
\end{tabular}

Legenda: ${ }^{1}$ Valores culturais, ${ }^{2}$ Idealismo e relativismo, ${ }^{3}$ Local de comprometimento, Identificação profissional e Motivação pública, ${ }^{4}$ Maquiavelismo e Emoções, ${ }^{5}$ Nacionalidade, ${ }^{6} \mathrm{Renda},{ }^{7} \mathrm{E}$ tnia, ${ }^{8} \mathrm{AM}$, MT, SP e RS, ${ }^{9} \mathrm{Ambiente}$ institucional, ${ }^{10}$ Valores éticos corporativos, ${ }^{11} \mathrm{Custos}$ e benefícios, ${ }^{12}$ Intensidade Moral.

Fonte: Dados da pesquisa. 
A variável individual gênero foi o enfoque mais pesquisado, tendo sido apresentada em 14 estudos, cujas amostras somadas totalizam 3.286 respondentes (Quadro 5). Em $46,7 \%$ dos estudos se verificou influência da variável sobre as decisões éticas, outros $57,1,7 \%$ concluíram que não há interveniência da variável. Tais resultados corroboram a pesquisa de O'Fallon e Butterfield (2005).

A variável idade foi pesquisada em 13 estudos, os quais totalizam 3.010 indivíduos, deste total, 38,5\% apresentaram influência significativa da variável com as decisões éticas e $61,5 \%$ concluíram que não há interveniência dessa variável na tomada de decisões éticas dos respondentes. Esses resultados, portanto, demonstram aderência aos do estudo de Kish-Gephart, Harrison e Trevino (2010) e aos de O'Fallon e Butterfield (2005).

A maturidade acadêmica foi pesquisada em 11 estudos, que totalizaram 2.375 respondentes. Em $72,7 \%$ destes, foram obtidas conclusões sobre a existência de influência significativa dessa variável nas decisões éticas, enquanto $27,3 \%$ concluíram que não ocorre influência. Esses resultados contrastam com os de O'Fallon e Butterfield (2005).

A variável experiência profissional foi abordada em nove pesquisas, cujas amostras totalizam 2.426 respondentes. Deste total, $55,6 \%$ das pesquisas concluíram pela influência da variável na decisão ética; $44,4 \%$ concluíram que não há relação significativa. $\mathrm{O}$ equilíbrio entre as conclusões positivas e negativas sobre a interveniência da experiência profissional na tomada de decisões éticas demonstra aderência aos achados de O'Fallon e Butterfield (2005).

Nove pesquisas da unidade de análise abordaram a influência de professar ou não alguma religião sobre as decisões éticas, e suas amostras somadas totalizaram 2.336 respondentes. Deste total, 55,6\% das pesquisas concluíram que a religiosidade dos respondentes não interfere nas suas decisões éticas, enquanto $44,4 \%$ delas concluíram que há relação da variável com a tomada de decisão ética dos indivíduos. Os achados desse estudo demonstram, portanto, aderência aos de Loe et al. (2000).

A influência da educação ética na tomada de decisões éticas dos respondentes, com base em 1.228 questionários respondidos, revela que há relação entre a educação ética e a tomada de decisões éticas pelos respondentes. Essa conclusão demonstra aderência aos achados de Abdolmohammadi,

Quadro 5 - Síntese dos estudos e o teste de hipótese
Fedorowicz e Davis (2009). Os estudos sobre a variável região tiveram 328 respondentes e seus dois resultados apresentaram $50 \%$ de correlação significativa. Os dois estudos que abordaram a variável estado civil (317 respondentes) revelam que esse aspecto não interfere nas decisões éticas.

O Código de Ética Profissional é a variável mais investigada entre os fatores situacionais, embora ainda seja abordado com menor frequência em relação aos fatores individuais. O Código de Ética Profissional é investigado em oito pesquisas, as quais totalizaram 3.598 respondentes, $87,5 \%$ concluíram que o conhecimento do CEPC e o seu reconhecimento como guia de conduta tem influência na tomada de decisões éticas dos respondentes. Em 12,5\% das pesquisas se verificou não ocorrer influência significativa do CEPC nas decisões éticas dos respondentes. Estes resultados são aderentes aos estudos de Loe et al. (2000), O'Fallon e Butterfield (2005) e Onyebuchi (2011).

A revisão sistemática apontou que os estudos em análise se dedicaram, predominantemente, às variáveis individuais, com destaque para o gênero, a idade, a maturidade acadêmica, a experiência profissional, a religiosidade e a educação ética, no entanto, não há consenso nos resultados para essas variáveis. No que se refere às variáveis situacionais, se destaca a frequência da abordagem do CEPC, com resultados consistentes sugerindo a relação desta variável com as decisões éticas. Quanto às variáveis relacionadas à questão ética, poucos estudos se dedicaram a sua investigação, no entanto, há consistência dos achados relacionados aos componentes da intensidade moral, conforme explicitados na teoria de Jones (1991).

\subsection{Metanálise}

Os estudos aptos a participarem da metanálise são aqueles que tinham base estatística para determinar se uma variável teve influência significativa $(\mathrm{p}<0,05)$ na tomada de decisão ética. Nesse sentido, foram selecionados 20 estudos que atendiam a essa condição (Quadro 5) e seus resultados foram analisados na tentativa de extrair se existem diferenças significativas na média dos resultados, ou seja, para cada variável individual e situacional foram estabelecidas as seguintes hipóteses: $\mathrm{H}_{0}=$ Não há diferenças significativas entre as médias; e $\mathrm{H}_{1}=$ Há diferenças significativas entre as médias das variáveis.

Continua...

\begin{tabular}{|l|l|l|l|l|l|l|}
\hline & \multicolumn{5}{|c|}{ Variáveis Individuais } & $\begin{array}{c}\text { Variáveis } \\
\text { situacionais }\end{array}$ \\
\hline Autor e ano & Gênero & Religião & $\begin{array}{l}\text { Experiência } \\
\text { Profissional }\end{array}$ & Maturidade & Idade & CEPC \\
\hline Assis, Friol e Tavares (2017) & Sim & & & & & \\
\hline Luczkiewicz (2015) & Não & Não & Não & Sim & Não & \\
\hline Lopes et al. (2006) & Sim & & & Sim & & \\
\hline Moraes, Silva e Carvalho (2010) & Sim & & Sim & & Sim & \\
\hline Silva (2011) & Sim & & Sim & & Sim & \\
\hline Clemente (2013) & Não & Não & & & Não & \\
\hline
\end{tabular}


Análise das Variáveis Intervenientes na Tomada de Decisões Éticas dos Profissionais e Estudantes de Ciências Contábeis Brasileiros

...continuação.

\begin{tabular}{|c|c|c|c|c|c|c|}
\hline Fajardo e Cardoso (2014) & Não & & & & Não & \\
\hline Milan (2014) & Não & Não & Sim & Sim & Não & \\
\hline Lucena et al. (2015) & Não & & & & Não & \\
\hline Costa (2016) & Não & & Não & Não & Não & \\
\hline Feil (2016) & Não & Sim & Não & Não & Não & Sim \\
\hline Ganassin (2016) & Não & Não & Não & & Sim & Não \\
\hline Mendonça e Gomes (2016) & Sim & Sim & & Sim & Não & \\
\hline Feil, Diehl e Schuck (2017) & Sim & Sim & Sim & Sim & Sim & \\
\hline Weiler (2016) & & Sim & & & Sim & \\
\hline Gomes (2012) & & & Sim & Sim & & Sim \\
\hline Barreto et al. (2011) & & Não & & & & \\
\hline Rosa e Feil (2017) & & & & & & Sim \\
\hline Alves et al. (2007) & & & & & & Sim \\
\hline Antonovz et al. 2010 & Sim & & & & & \\
\hline Amostra (respondentes) & 3.998 & 2.336 & 2.426 & 1.977 & 2.795 & 3.416 \\
\hline Teste de hipótese (Qui-quadrado & 1,000 & 0,819 & 0,819 & 0,247 & 0,165 & 0,002 \\
\hline
\end{tabular}

Fonte: Dados da pesquisa.

O teste não paramétrico Qui-quadrado revela que, entre as variáveis submetidas, a única cujo valor de $p<0,05 \mathrm{e}$, portanto, tem relação estatisticamente significativa com a tomada de decisão ética é o conhecimento a respeito do CEPC, ou seja, neste caso se rejeita a $\mathrm{H}_{0}$. As variáveis individuais (gênero, idade, maturidade, experiência profissional, e religião) apresentaram um valor de $p>0,05$, sendo assim, se aceita a $\mathrm{H}_{0}$, pois não apresentaram diferenças significativas.

A revisão sistemática identificou 28 estudos conduzidos no Brasil sobre o tema, abrangendo 7.580 respondentes de 22 Estados das cinco regiões geográficas do país, ao longo do período entre os anos de 2006 e 2017. Tal abrangência demonstra que o interesse pelo tema da tomada de decisão ética dos profissionais e estudantes de Ciências Contábeis é crescente entre pesquisadores e instituições de todo o país. A elevada titulação acadêmica da maioria dos autores e a publicação dos estudos em periódicos dos estratos superiores da classificação Qualis, além de evidenciar o interesse pelo tema, aumentam a credibilidade relativa à qualidade científica dos estudos.

Quanto à frequência de abordagem das variáveis, este estudo verificou que as pesquisas nacionais se dedicam, predominantemente, às variáveis individuais, em comparação às variáveis situacionais e às relativas à questão ética. Isto pode se dever, entre outras razões, ao fato de que essas são mais diretamente verificáveis e mensuráveis em um survey do que as que envolvem alguns dos fatores situacionais ou as ligadas à questão ética.

As variáveis individuais investigadas com maior frequência nos estudos em análise foram: o gênero $(53,6 \%)$, a idade $(46,4 \%)$, a maturidade acadêmica $(39,3 \%)$, a experiência profissional $(32,1 \%)$ e a religiosidade $(32,1 \%)$. Estudos correlatos também apresentam o gênero como a variável mais frequente (LOE et al., 2000; O'FALLON; BUTTERFIELD, 2005).

A seguir, as variáveis que se destacaram na revisão e na metanálise pela frequência de abordagem e pelos resultados obtidos são discutidas individualmente. Para tanto, inicia- se pelos comentários relativos às variáveis individuais, seguindo-se as situacionais e, posteriormente, as relativas à questão ética.

A influência do gênero na tomada de decisões éticas não ficou comprovada através do conjunto dos estudos em análise. A revisão sistemática demonstrou equilíbrio entre os estudos, que encontraram relação entre a variável e a decisão ética e os que concluíram que esta relação não ocorre. $\mathrm{O}$ teste Qui-Quadrado reforçou esse resultado, ao indicar a retenção da hipótese nula para a variável. Esses achados refletem as conclusões de Loe et al. (2000) e O'Fallon e Butterfield (2005). Os resultados obtidos para o gênero parecem reforçar a afirmação de Kish-Gephart, Harrison e Trevino (2010), de que a relação entre a variável e a tomada de decisão ética ainda não está esclarecida.

A maioria dos estudos concluiu não haver relação da idade com a tomada de decisões éticas. O teste Qui-Quadrado confirmou esse achado por meio da aceitação da hipótese nula e corrobora com a revisão de literatura de O'Fallon e Butterfield (2005).

Na maturidade acadêmica, embora a maioria dos estudos em análise aponte correlação da variável com a tomada de decisão ética, o teste Qui-Quadrado indicou a aceitação da $\mathrm{H}_{0}$. Assim, a maturidade acadêmica pode servir, por um lado, como a oportunidade de compreender a própria responsabilidade pelas consequências das atitudes éticas ou antiéticas, ou por outro, como a possibilidade de obter o conhecimento necessário para identificar e obter possíveis vantagens indevidas.

A experiência profissional apresenta um equilíbrio entre as conclusões positivas e negativas para essa variável. O teste Qui-Quadrado sugere a aceitação da hipótese nula. Deve-se ressaltar que a experiência profissional é, necessariamente, permeada pelas interações com os fatores situacionais relacionados ao ambiente de trabalho, os quais foram apresentados nos modelos de Hunt e Vitell (2006) e Trevino (1986).

A religião aponta um equilíbrio entre os resultados que 
afirmam e os que negam a influência dessa variável e, nessa mesma lógica, o Qui-Quadrado indicou a validade da hipótese nula, ou seja, a de que não ocorre relação estatisticamente significativa entre professar uma religião e tomar decisões mais ou menos éticas. Nessa lógica, corrobora com Loe et al. (2000) e discorda de O'Fallon e Butterfield (2005). No entanto, os autores concluíram que os respondentes, ainda que professem religiões, não necessariamente estabelecem relações entre os valores religiosos que seguem em suas vidas pessoais e as suas decisões na prática da profissão contábil.

A educação ética sugere uma relação positiva com a tomada de decisões éticas dos estudantes e profissionais. Embora apenas três desses estudos tenham apresentado testes estatísticos e, portanto, a variável não tenha sido selecionada para a metanálise, destaca-se o consenso dos resultados positivos. Esse consenso reforça a utilidade da educação ética na formação dos futuros profissionais contábeis.

O Código de Ética se apresenta como um fator que leva à tomada de decisões mais éticas pelos indivíduos. Essa conclusão foi confirmada pelo teste Qui-Quadrado, o qual indicou a rejeição da hipótese nula relativamente a essa variável. Esses resultados aderem aos dos estudos de Loe et al. (2000) e de O'Fallon e Butterfield (2005).

Assim, verifica-se que a investigação sobre a relação desse fator com a tomada de decisão ética tem, consistentemente, apresentado resultados positivos ao longo do tempo e em diversos países. Tal consistência de resultados demonstra a utilidade e a necessidade da abordagem do Código no ensino da profissão, de modo a permitir aos estudantes o conhecimento aprofundado desse instrumento, o qual pode vir a subsidiar muitas de suas futuras decisões profissionais.

O crescente interesse e a consistência dos achados recentes para a variável intensidade moral em pesquisas fora do Brasil levam a considerar a possibilidade de expansão das pesquisas, que testem essa variável e seus componentes no Brasil.

\section{Conclusão}

Os recentes casos de fraudes e escândalos corporativos envolvendo profissionais da contabilidade promoveram o aumento do interesse do público sobre o papel desses profissionais nas condutas ilícitas. O campo de estudos da ética profissional tem se dedicado à investigação dos fatores, que levam os profissionais de diversas áreas, inclusive a contábil, a agir mais ou menos eticamente. No entanto, os resultados dos estudos não demonstram consenso sobre quais variáveis determinam a decisão e a conduta ética dos profissionais.

Nesse contexto, este estudo objetivou uma análise dos fatores que influenciam na tomada de decisões éticas dos profissionais e estudantes de contabilidade brasileiros. Para tanto, foram identificados, por meio da revisão sistemática, 28 estudos conduzidos no Brasil e dedicados à investigação das variáveis intervenientes na decisão ética, totalizando 7.580 respondentes, entre profissionais e estudantes de Ciências Contábeis de 22 Estados das cinco regiões do país, entre 2006 e 2017 .

A revisão sistemática demonstrou que há predomínio de pesquisas sobre as variáveis individuais. Entre essas, o gênero, a idade, a religiosidade e fatores relacionados à experiência acadêmica e de trabalho se destacam como os mais frequentes. Entretanto, não foi verificado consenso dos resultados sobre a influência ou não dessas variáveis, à exceção da educação ética, cuja influência foi verificada em todos os estudos que a abordaram. Entre os fatores situacionais, a maior frequência de abordagem entre os estudos revisados foi do Código de Ética Profissional, com resultados consistentes indicando a relação da variável com a decisão ética. A variável intensidade moral da questão ética, embora ainda seja emergente como um fator de interesse nas pesquisas, tem indicado relação positiva com a decisão ética.

A metanálise revela que as variáveis individuais e situacionais submetidas ao teste foram indicadas a rejeição da hipótese nula, isto é, foi apontada relação estatisticamente significativa apenas para o Código de Ética Profissional. Portanto, resultados sugerem que a educação ética, bem como o Código de Ética são entre as variáveis investigadas no Brasil até o momento, as que têm produzido os resultados mais consistentes. Esses achados demonstram a importância da educação ética e do estudo aprofundado do CEPC na formação dos futuros profissionais contábeis. O papel de outras variáveis, como: o gênero, a idade, a religiosidade e a experiência profissional, parece ainda necessitar de maior esclarecimento. Os componentes da intensidade moral da questão ética, embora sejam fatores de investigação ainda incipiente, também parecem merecer maior investigação.

Considerando que o interesse sobre as variáveis intervenientes na decisão ética ainda está se ampliando no país, conforme demonstra a evolução do número de pesquisas, ao longo dos anos, podem ser apontadas novas perspectivas para essa investigação. Além do aprofundamento da investigação sobre a intensidade moral, recomenda-se a expansão da pesquisa sobre a influência de fatores situacionais, além do Código de Ética e de fatores individuais menos investigados até o momento.

As limitações deste estudo vão ao encontro da possibilidade de haver periódicos não indexados nas bases consultadas e que, também, não tenham sido relacionados nas referências bibliográficas das pesquisas localizadas. Além disso, os resultados deste estudo não podem ser generalizados, visto que as pesquisas da unidade de análise, além de serem em número reduzido, se utilizaram de diferentes procedimentos e instrumentos de coleta de dados.

\section{Referências}

ABDOLMOHAMMADI, M.J.; FEDOROWICZ, J.; DAVIS, O. Accountants' cognitive styles and ethical reasoning: a comparison across 15 years. J. Accounting Educ., v.27, p.185-196, 2009. doi: 10.1016/j.jaccedu.2010.07.003.

ALVES, F.J.S. A adesão do contabilista ao código de ética da 
sua profissão: um estudo empírico sobre percepções. São Paulo: Universidade de São Paulo. 2005.

ALVES, F.J.S. et al. Um estudo empírico sobre a importância do código de ética profissional para o contabilista. Rev. Contab. Finanças, v.18, p.58-68, 2007. doi: 10.1590/S151970772007000300006 .

ALVES, L.L. O código de ética e os profissionais da contabilidade: estudo de caso na cidade de Dourados/MS. Campo Grande: Universidade Federal da Grande Dourados, 2010.

ANTONOVZ, T. et al. Atitudes éticas dos contadores: evidências recentes de uma pesquisa com alunos e profissionais contábeis sob perspectiva de gênero. Rev. Contab. Organizações, v.4, n.10, p.87-105, 2010. doi: 10.11606/rco.v4i10.34778.

ANZEH, B.A.; ABED, S. The extent of accounting ethics education for bachelor students in Jordanian universities. $J$. Management Res., v.7, n.2, p.121-143, 2015. doi: 10.5296/jmr. v7i2.6957.

ASSIS, P.P.S.; FRIOL, M.V.; TAVARES, M.F.N. Earnings management: julgamento ético dos profissionais contábeis do Brasil.In: ENCONTRO NORDESTINO DE CONTABILIDADE, Natal, 4. 2017. Anais... Rio Grande do Norte-Natal, 2017.

BARRETO, I.G. et al. Um estudo avaliativo das decisões dos contabilistas no estado de Pernambuco sob a perspectiva dos modelos éticos contemporâneos: uma abordagem quanto a religião. ReCont Reg. Contábil, v.2, n.1, p.55-76, 2011.

BBCBrasil. ENRON: perguntas e respostas. 2002. Disponível em: www.bbc.com/portuguese/economia/020128_esp_eronqa shtml\#top. Acesso em: 19 out. 2019.

BBCBrasil. ÓRGÃO regulador processa KPMG por fraude nos EUA. 2003. Disponível em: www.bbc.com/portuguese/ economia/030130 kpmgdb.shtml. Acesso em: 19 out. 2019

BRANCHER, C.; NEU, M.A.; BOFF, M.L. Ética profissional: entendimento dos acadêmicos de ciências contábeis da Unoesc. Unoesc Ciênc. ACSA, v.1, n.1, p.31-38, 2010.

BISQUERRA, R.; SARRIERA, J.C.; MARTÍNEZ, F. Introdução à estatística: enfoque informático com o pacote estatístico SPSS. Porto Alegre: Artmed, 2004.

CLEMENTE, L.S. Fatores determinantes do julgamento ético dos profissionais da contabilidade no Brasil. São Paulo: Universidade Presbiteriana Mackenzie, 2013.

CONNELLY, S.; HELTON-FAUTH, W.; MUMFORD, M.D. A managerial in-basket study of the impact of trait emotions on ethical choice. J. Business Ethics, v.51, n.3, p.245-267, 2004. doi: 10.1023/B: BUSI. 0000032494.51162.d3.

COSTA, A.P.P. Casos de fraudes corporativas financeiras: antecedentes, recursos substantivos e simbólicos relacionados. São Paulo: Fundação Getúlio Vargas, 2011.

COSTA, M. Ética e moral no processo de tomada de decisão: o caso dos alunos de ciências contábeis. Curitiba: Universidade Tecnológica Federal do Paraná, 2016.

CULLINAN, C. et al. Organization-harm vs. organizationgain ethical issues: an exploratory examination of the effects of organizational commitment. J. Bus. Ethics, v.80, n.2, p.225-235, 2008. doi: 10.1007/s10551-007-9414-4.

CURTIS, M.B. Are audit-related ethical decisions dependent upon mood? J. Bus. Ethics, v.68, n.2, p.191-209, 2006. doi: 10.1007/s10551-006-9066-9.

ELY, L. Escândalos contábeis são alvo de investigação. 2010. Disponível em: jcrs. uol.com.br/site/noticia.php? codn=49352. Acesso em: 20 abr. 2019.
FAJARDO, B.A.G.; CARDOSO, R.L.A ocasião faz a denúncia? uma abordagem multinível para os contabilistas brasileiros. Braz. Bus. Rev., v.11, n.5, p.25-50, 2014.

FEIL, A.A. Análise das variantes na tomada de decisão ética do profissional contábil. In: CONGRESSO BRASILEIRO DE CONTABILIDADE. Fortaleza, 11 a 14 set. de 2016. Anais... Fortaleza, 2016.

FEIL, A.A.; DIEHL, L.; SCHUCK, R.J. Ética profissional e estudantes de contabilidade: análise das variáveis intervenientes. Cad. EBAPE.BR, v.15, n.2, p.256-273, 2017. doi 10.1590/1679395160994.

FERRELL， O.C.; FRAEDRICH，J.; FERRELL， L. Ética empresarial: dilemas, tomada de decisões e casos. Rio de Janeiro: Ernesto Reichmann, 2001.

FIELD, A. Descobrindo a estatística usando o SPSS. Porto Alegre: Artmed, 2009.

FILGUEIRAS, M. L. Escândalo da Petrobras põe os auditores na berlinda. Exame. 2015. Disponível em: exame.abril.com.br/ revista-exame/dava-pra-pegar/. Acesso em: 20 abr. 2018.

GANASSIN, E.J.F. Investigação de variáveis que influenciam a ética profissional contábil: uma aplicação das teorias dos estímulos internos e externos. Brasília: UnB, 2016.

GOMES, M.S. A difícil função de ser ético: a importância do código de ética no exercício da profissão contábil. João Pessoa: Universidade Estadual da Paraíba, 2012.

HORTA, A.M. Terremoto global. 2002. Disponível em: http:// revistaepoca.globo.com/Epoca/0,6993,EPT344659-1663-1,00. html. Acesso em: 19 out. 2018.

HUNT, S. D.; VITELL, S. J. A general theory of marketing ethics. J. Macromarketing, v.6, p.5-16, 1986. doi: 10.1177/0276146706290923.

JONES, T.M. Ethical decision making by individuals in organizations: an issue-contingent model. Acad. Manag. Rev, v.16, n.2, p.366-395, 1991. doi: 10.2307/258867.

KELIN, J.M. A percepção dos alunos de ciências contábeis da Universidade de Caxias do Sul-campus sede - sobre o código de ética profissional do contador. Caxias do Sul: Universidade de Caxias do Sul, 2014.

KISH-GEPHART, J.J.; HARRISON, D.A.; TREVINO, L.K. Bad apples, bad cases, bad barrels: meta-analytic evidence about sources of unethical decisions at work. J. Appl. Psychol., v.95, n.1, p.1-31, 2010. doi: 10.1037/a0017103.

KOHLBERG, L. Stage and sequence: the cognitivedevelopmental approach to socialization. In: GOSLIN, D. (Org.). Handbook of socialization theory and research. Chicago: Rand McNally, 1969. p.347-480.

LOPES, J.E.G. et al. Uma análise avaliativa de atitudes de estudantes de ciências contábeis e dilemas éticos sob uma perspectiva de gênero, maturidade acadêmica e ambiente institucional. Ensaio Aval. Pol. Públ. Educ., v.14, n.51, p.209$222,2006$.

LUCENA, E.R.F.C.V. et al. Ética: a tendência de os profissionais contábeis denunciarem atos questionáveis. Rev. Contab. Org., v.24, p.4-17, 2015. doi: 10.11606/rco.v9i24.55421.

LUCZKIEWICZ, D.A. Desenvolvimento moral e valores culturais dos estudantes de graduação em ciências contábeis no Brasil. 2015. 103f. Dissertação (Mestrado) - Fundação Escola de Comércio Álvares Penteado - FECAP, 2015.

LUPION, B. Por que as auditorias dificilmente detectam corrupção em uma empresa. 2017. Disponível em: https://www. 
nexojornal.com.br/expresso/2016/07/27/Por-que-as-auditoriasdificilmente-detectam-corrup $\% \mathrm{C} 3 \% \mathrm{~A} 7 \% \mathrm{C} 3 \% \mathrm{~A} 3$ o-em-umaempresa. Acesso em: 12 set. 2018.

MEDEIROS, A.W.; VIEIRA, E.R.F.C. Percepção comparativa no âmbito da ética entre os alunos do curso de ciências contábeis do $1^{\circ}$. e $4^{\circ}$. ano da FARN. Interface, v.7, n.1, p.93-108, 2010.

MENDONÇA, W.S.; GOMES, G.S. Análise das concepções dos estudantes do curso de ciências contábeis acerca da ética. Rev. Auditoria, Governança Contab., v.4. n.15, p.45-60, 2016.

MILAN, J.C. Comportamento oportunista nas escolhas contábeis: a influência das emoções e do traço de personalidade. São Paulo: FECAP, 2014.

MORAES, M.C.C.; SILVA, A.M.C.; CARVALHO, F.A.A. O comportamento dos futuros contabilistas perante diferentes dilemas éticos. Pensar Contábil, v.12, n.48, p.22-30, 2010.

O'FALLON, M.J.; BUTTERFIELD, K.D.A review of the empirical ethical decision-making literature: 1996-2003. J. Bus. Ethics, v.59, n.4, p.375-413, 2005. doi: 10.1007/s10551-0052929-7.

ONYEBUCHI, V.N. Ethics in accounting. Int. J. Bus. Soc. Sci., v.2, n.10, p.275-276, 2011.

RAMACCIOTTI, C. A ética e a confiança nas organizações: um estudo descritivo junto a profissionais da Grande São Paulo. São Paulo: Fundação Getúlio Vargas, 2007.

RODRIGUES, C.L. Metanálise: um guia prático. Porto Alegre: Universidade Federal do Rio Grande do Sul, 2010.

ROSA, F.S.; FEIL. A.A.A percepção sobre ética dos estudantes em ciências contábeis. Rev. Cient. Tecnol. Gestão Hum., v.7, n.2, p.20-37, 2017.

SÁ, A.L. Ética Profissional. São Paulo: Atlas, 2012.

SAMPAIO, R.E.; MANCINI, M.C. Estudos de revisão sistemática: um guia para a síntese criteriosa da evidência científica. Rev Bras Fisioter, v.11, n.1, p.83-89, 2007. doi: 10.1590/S1413-35552007000100013.

SAMPIEIRI, H.R.; COLLADO, C.F.; LUCIO, M.P. Metodologia de pesquisa. Porto Alegre: Penso, 2013.

SANTOS, L.C.B. et al. Gerenciamento de resultados visto sob o aspecto ético. In: SEMEAD - SEMINÁRIOS EM ADMINISTRAÇÃO, São Paulo, 2014. Anais ... São Paulo, 2014.

SILVA, A.R.N.; FIGUEIREDO, S.M.A. Desenvolvimento da percepção sobre ética profissional entre estudantes de contabilidade. Rev. Bras. Contabilidade, n.165, p.27-39, 2007.

SILVA, Z.Y.B. Julgamento ético-ideológico dos estudantes de contabilidade de São Paulo. São Paulo: Universidade Presbiteriana Mackenzie, 2011.

SORENSEN, D.P.; MILLER, S.E.; CABE, K.L. Developing and measuring the impact of an accounting ethics course that is based on the moral philosophy of Adam Smith. J. Business Ethics, v.140, n.175, p.1-17, 2015. doi: 10.1007/s10551-015-2656-7.

SOUZA, A.P. et al. O código de ética como instrumento para a tomada de decisão: um estudo sobre a percepção do contador acerca da ética profissional. Rev. Adm. Contab. Fac. Estácio Pará, v.4, n.8, p.28-52, 2017.

TRENTIN, G.N.S.; DOMINGUES, M.J.C.S.; CASTRO, D.R.M.S. Percepção dos alunos de ciências contábeis sobre ética profissional. In: CONGRESSO BRASILEIRO DE CUSTOS, 15, 2008. Curitiba. Anais... Curitiba, 2008.

TREVINO, L.K. Ethical decision making in organizations: a person-situation interactionist model. Academy Manag. Rev., v.11, n.3, p.601-617, 1986. doi 10.2307/258313.

WEILER, P. F. Análise das variáveis intervenientes relacionadas à ética dos profissionais contábeis. Lajeado: Centro Universitário Univates, 2016. 